\title{
USP14 activation promotes tumor progression in hepatocellular carcinoma
}

\author{
GANG HUANG ${ }^{1 *}$, LIMEI $\mathrm{LI}^{2,3^{*}}$ and WEIPING ZHOU ${ }^{1}$ \\ ${ }^{1}$ Department of Surgery, Eastern Hepatobiliary Surgery Hospital, Second Military Medical University; \\ ${ }^{2}$ Clinical and Translational Research Center Shanghai East Hospital, Key Laboratory of Arrhythmias \\ of Ministry of Education; ${ }^{3}$ Tongji University School of Medicine, Shanghai, P.R. China
}

Received April 12, 2015; Accepted July 6, 2015

DOI: $10.3892 /$ or.2015.4296

\begin{abstract}
To elucidate the molecular mechanisms underlying the pathogenesis and treatment of human primary hepatocellular carcinoma (HCC), it is important to explore novel $\mathrm{HCC}$-associated genes. In the present study, we examined the expression of ubiquitin-specific peptidase 14 (USP14) in patients with HCC using quantitative PCR and immunohistochemical techniques. The expression of USP14 in tumor tissues of patients with HCC was significantly higher than that in adjacent non-cancerous and normal liver tissues. It was also determined whether the expression profile of USP14 was associated with the clinical characteristics of HCC. Increased USP14 expression was associated with some clinicopathological variables, such as advancing tumor stage. A Kaplan-Meier curve analysis demonstrated that patients with HCC having a high USP14 expression had a significantly poorer prognosis after surgery than patients with lower USP14 expression levels. Knockdown of USP14 with the lentiviral vector delivery of shRNA in human hepatocarcinoma SMMC7721 cells suppressed cell proliferation, altered the cell cycle and induced cell apoptosis. Additionally, the
\end{abstract}

Correspondence to: Professor Weiping Zhou, Hepatic Surgery Department III, Eastern Hepatobiliary Surgery Hospital, Second Military Medical University, 225 Changhai Road, Shanghai 200438, P.R. China

E-mail: ehphwp@126.com

*Contributed equally

Abbreviations: BCA, bicinchoninic acid; CCK-8, cell counting kit-8; DFS, disease-free survival; DUB, deubiquitinating enzymes; FACS, fluorescence-activated cell sorting; FDA, food and drug administration; HCC, hepatocellular carcinoma; IHC, immunohistochemistry; IRSs, immunoreactive scores; MCL, mantle cell lymphoma; MM, multiple myeloma; NSCLC, non-small cell lung cancer; UPS, ubiquitin proteasome system; USP14, ubiquitin-specific peptidase 14

Key words: USP14, hepatocellular carcinoma, prognosis, therapeutics, tumor recurrence
Wnt/ $\beta$-catenin pathway was activated in HCC patients with USP14 overexpression. These findings strongly suggested that USP14 activation plays an oncogenic role in promoting tumor progression in HCC. Thus, our findings suggested that USP14 is involved in the progression of HCC and may be a useful therapeutic target in HCC. These findings likely reflect the key role that USP14 plays in the pathogenesis of HCC. Therefore, the identification of USP14 and USP14-driven genes may promote the investigation of its functional role to develop more effective therapies for HCC, especially advanced HCC.

\section{Introduction}

As one of the most common malignant tumors, human primary hepatocellular carcinoma (HCC) is the third leading cause of cancer-related mortality worldwide $(1,2)$, with the incidence increasing, especially in East Asia and South Africa. Approximately $50 \%$ of patients worldwide with HCC are located in China, where HCC is the second leading cause of cancer-related mortalities (3-5). Although marked progress has been made in the treatment of $\mathrm{HCC}$, many challenges still remain, such as the difficulty of early diagnosis (6), the high rate of metastasis when recurrence develops (7) and the lack of an effective therapeutic target (8-10). Hepatic resection is a well accepted therapy for $\mathrm{HCC}$, however, many patients are diagnosed with advanced HCC and are thus unable to undergo surgery $(11,12)$. Alternative treatments do not substantially improve patient prognosis when HCC is deemed unresectable. Therefore, studies have been conducted to identify novel genes and proteins associated with the malignant proliferation of tumor cells in the pathogenesis of HCC to develop more effective therapies.

The investigation of molecular mechanisms leading to HCC development and progression is necessary to identify new targets for early diagnosis and treatment (8). To gain new insight into the molecular mechanisms underlying the pathogenesis of HCC, we searched for HCC-specific molecules by screening genes that are differentialy expressed between cancer and non-cancer counterparts of the liver and identified a novel HCC-associated gene. We found that ubiquitin-specific peptidase 14 (USP14), encoding an $\sim 56 \mathrm{kDa}$ cytoplasmic protein $(13,14)$, was upregulated in the liver of patients with HCC. USP14 belongs to the family of deubiquitinating 
enzymes (DUB) composed of His and Cys domains. USP14 removes the (poly)ubiquitin moiety from protein substrates in a particular form, as it is activated catalytically following specific association with the $19 \mathrm{~S}$ regulatory elements of the 26S proteasome (15).

Dysfunction of the ubiquitin proteasome system (UPS) is involved in numerous diseases, including cancer. The primary means of selective intracellular protein degradation is through proteasomal degradation after ubiquitin modification (16). Ubiquitination of proteins is a dynamic balanced process. DUBs can reverse the ubiquitination reaction because the deubiquitinating enzyme removes the ubiquitin tag from a target protein that has been modified by ubiquitin polymers $(17,18)$. To maintain intracellular protein stability and homeostasis, the UPS is precisely regulated. Some studies have reported that DUBs can regulate cell cycle-associated proteins by deubiquitination to maintain the stability of these intracellular proteins, for example, cyclin-dependent kinase inhibitors CDKN1A and CDKN1B (19) and tumor-suppressor gene P53 (20). This influences the cell cycle as well as cell proliferation. USP1 (21), USP9X (22), USP28 (23) and USP44 (24) have been reported to play important roles in the development and progression of cancer. As vital regulators of protein degradation, DUBs have also become potential targets in drug design as inhibitors of malignant disease.

Recent studies suggest a high expression of USP14 in some tumors, such as in colorectal cancer $(25,26)$, epithelial ovarian cancer (27), lung adenocarcinoma (28) and intrahepatic cholangiocarcinoma (29). Therefore, in the present study, we examined the expression of USP14 in HCC. The biological role of USP14 activation in the progression of HCC was investigated and the association between the expression profile of USP14 and clinical characteristics of HCC was also analyzed to explore the mechanisms regulating HCC initiation and progression and to determine whether USP14 may be a novel target in the diagnosis or treatment of HCC.

\section{Materials and methods}

Patients and tissue samples. Formalin-fixed and paraffinembedded liver tumor specimens and matched tumor-adjacent normal tissues were obtained for immunohistochemical analysis from 31 consecutive patients diagnosed with HCC in the Eastern Hepatobiliary Surgery Hospital (30), the Second Military Medical University, Shanghai, China, from 2004 to 2007. To confirm the expression level of USP14, stained sections were evaluated according to the German immunoreactive scoring system using immunoreactive scores (IRSs) as previously described $(31,32)$. Briefly, IRSs were assigned sub-scores for immunoreactivity intensity (0-3) and distribution $(0-4)$. These sub-scores were then added to obtain the final IRS score. The sub-scores for immunoreactive staining intensity were defined as: 0 , no staining; 1 , weakly stained; 2 , moderately stained and 3 , strongly stained. The sub-scores for immunoreactivity distribution were defined as: $0,<5 \%$, $1,5-25 \%, 2,25-50 \%, 3,50-75 \%$ and $4,>75 \%$. Fresh-frozen liver tissues from 31 patients with HCC and normal liver tissues from 6 patients who underwent primary hepatectomy were used for RNA extraction and quantitative PCR (qPCR) assays. For the 31 patients with HCC, 26 had been followed for 3 years and their complete clinical data were electronically recorded. The disease-free survival rate was defined as the interval between the dates of surgery and recurrence, and where recurrence was not diagnosed, patients were censored based on the dates of mortality or the last follow-up. The present study was performed in accordance with the ethical standards of the Human Experimentation of the Second Military Medical University.

RNA isolation and cDNA synthesis. Total RNA was isolated from fresh-frozen HCC tumor specimens, healthy control tissues and cell lines using TRIzol (Invitrogen-Life Technologies, Carlsbad, CA, USA) according to the manufacturer's instructions. The extracted RNA samples $(2 \mu \mathrm{g})$, which were kept at $-80^{\circ} \mathrm{C}$, were reverse-transcribed into cDNA using M-MLV reverse transcriptase (Promega, Madison, WI, USA) in a final reaction volume of $20 \mu \mathrm{l}$. Reverse transcription of total RNA was performed using random hexamers (Roche Diagnostics, Penzberg, Germany).

qPCR analysis. For qPCR amplification, SYBR-Green (Takara, Otsu, Japan) and $0.5 \mu \mathrm{l}$ of cDNA per reaction were used. Primers of human USP14 were: forward, 5'-GGCTTC AGCGCAGTATATTA-3' and reverse, 5'-CAGATGAGG AGTCTGTCTCT-3'. The primers were synthesized by Sangon (Shanghai, China). Reactions were performed under the following conditions: $94^{\circ} \mathrm{C}$ for $6 \mathrm{~min} ; 45$ cycles of $94^{\circ} \mathrm{C}$ for $20 \mathrm{sec}, 60^{\circ} \mathrm{C}$ for $31 \mathrm{sec}, 72^{\circ} \mathrm{C}$ for $32 \mathrm{sec}$ and $72^{\circ} \mathrm{C}$ for $10 \mathrm{~min}$. Amplification and detection of SYBR-Green were performed with a GeneAmp PCR System 5700 (Applied Biosystems). The human $\beta$-actin gene was used as an internal control. Threshold changes relative to normal liver controls were determined using cycle $(\mathrm{Ct})$ values from triplicate reactions and averaged. Fold was measured using the $2^{-\Delta \Delta C T}$ method.

Western blot analysis of USP14. The tissues or cells were lysed in RIPA buffer containing a protease inhibitor (Roche Applied Science, Indianapolis, IN, USA) and homogenated. After incubation for $20 \mathrm{~min}$ at $4^{\circ} \mathrm{C}$ and centrifugation at $10,800 \mathrm{x} \mathrm{g}$ for $25 \mathrm{~min}$ at $4^{\circ} \mathrm{C}$, the supernatant was collected. Protein concentrations were estimated using the bicinchoninic acid (BCA) assay (Sango, China). For the western blot analysis, proteins were loaded onto 10\% SDS-PAGE gels and transferred to polyvinylidene difluoride membranes. After transfer, the membranes were incubated with a USP14 primary antibody (Santa Cruz Biotechnology, Inc., CA, USA) for $1 \mathrm{~h}$ at room temperature or overnight at $4^{\circ} \mathrm{C}$ on a shaker, followed by a horseradish peroxidase-conjugated secondary antibody (Beyotime, Shanghai, China). Protein bands were detected with ECL Plus reagents (Amersham Biosciences, Switzerland).

Transfection. SMMC7721 cells were seeded in 6-well plates $\left(4 \times 10^{3}\right.$ cells/well $)$ in $0.5 \mathrm{ml}$ of complete growth medium. On the day of transfection, cell density was 50-80\% confluent. Suspensions of three USP14 shRNA lentiviruses (USP14-siRNA1, USP14-siRNA2 and USP14-siRNA3) were added to different plates of SMMC7721 cells and incubated for $3 \mathrm{~h}$ at $37^{\circ} \mathrm{C}$. Fresh medium $(2 \mathrm{ml})$ was then added. After $24 \mathrm{~h}, 0.5 \mathrm{ml}$ of complete growth medium was replaced with growth medium. 
Cell proliferation assay. Human SMMC7721 hepatocarcinoma cells $\left(4 \times 10^{3}\right.$ cells/well) with various treatments were seeded on a 96-well plate in DMEM (high glucose) supplemented with $10 \%$ fetal bovine serum and $1 \%$ antibiotic/antimycotic solution (Sigma-Aldrich, St. Louis, MO, USA). Cell proliferation was detected over 5 days using the Cell Counting kit-8 (CCK-8) assay according to the manufacturer's instructions.

Apoptosis analysis and clonogenic assays. Apoptosis was monitored with an Annexin V-FITC apoptosis detection kit (Invitrogen-Life Technologies) according to the manufacturer's instructions. SMMC7721 cells with various treatments were seeded in 6 -well plates $\left(4 \times 10^{3}\right.$ cells/well) for clonogenic assays. After growing for 10 days, the number of colonies was counted. A colony was defined as cell clusters with $\geq 50$ cells.

Cell cycle assay. SMMC7721 cells with various treatments were incubated with propidium iodide (PI; Beyotime), according the manufacturer's instructions. The samples were examined using a fluorescence-activated cell sorting (FACS) assay and the results were analyzed with CellQuest software (both from Becton-Dickinson, Franklin Lakes, NJ, USA).

Statistical analysis. Survival curves were estimated using the Kaplan-Meier method and the differences were compared with the log-rank test. Statistical Package for the Social Sciences 15.0 software (SPSS Inc., Chicago, IL, USA) was used for the statistical analyses and $\mathrm{P}<0.05$ was considered significant. The Pearson's $\chi^{2}$ test or Fisher's exact test was used to analyze the relationship between USP14 expression and the HCC clinicopathological characteristics. Data are presented as means \pm SE. Other data were analyzed with one-way ANOVA. $\mathrm{P}<0.05$ was considered significant.

\section{Results}

USP14 expression in HCC specimens. To determine the expression level of USP14 in hepatocellular carcinoma specimens, we detected USP14 expression levels using RT-qPCR and immunohistochemistry (IHC) in livers obtained from patients with HCC following liver resection. The RNA was extracted from 31 cases of HCC specimens and 6 normal livers. The USP14 mRNA expression was markedly increased in 26 of $31(83.87 \%)$ HCC tissues compared with that in normal liver tissues (Fig. 1A). We also examined the protein expression of USP14 using IHC staining in $31 \mathrm{HCC}$ tumor tissues and matched tumor-adjacent normal tissues. A high USP14 protein expression was detected in the malignant cells, whereas weak staining was observed throughout the matched normal controls (Fig. 1B). USP14 expression was observed in $83.87 \%$ of HCC specimens (26/31) and the immunoreactivity was predominantly localized to the cytoplasm of the malignant cells.

Association of USP14 expression with clinicopathological characteristics. We evaluated the clinical significance of the USP14 expression in HCC in high and low USP14-expressing groups based on the results of the expression levels of the USP14 protein. Thus, patients were placed into a group with high intratumoral USP14 protein density or a group with low
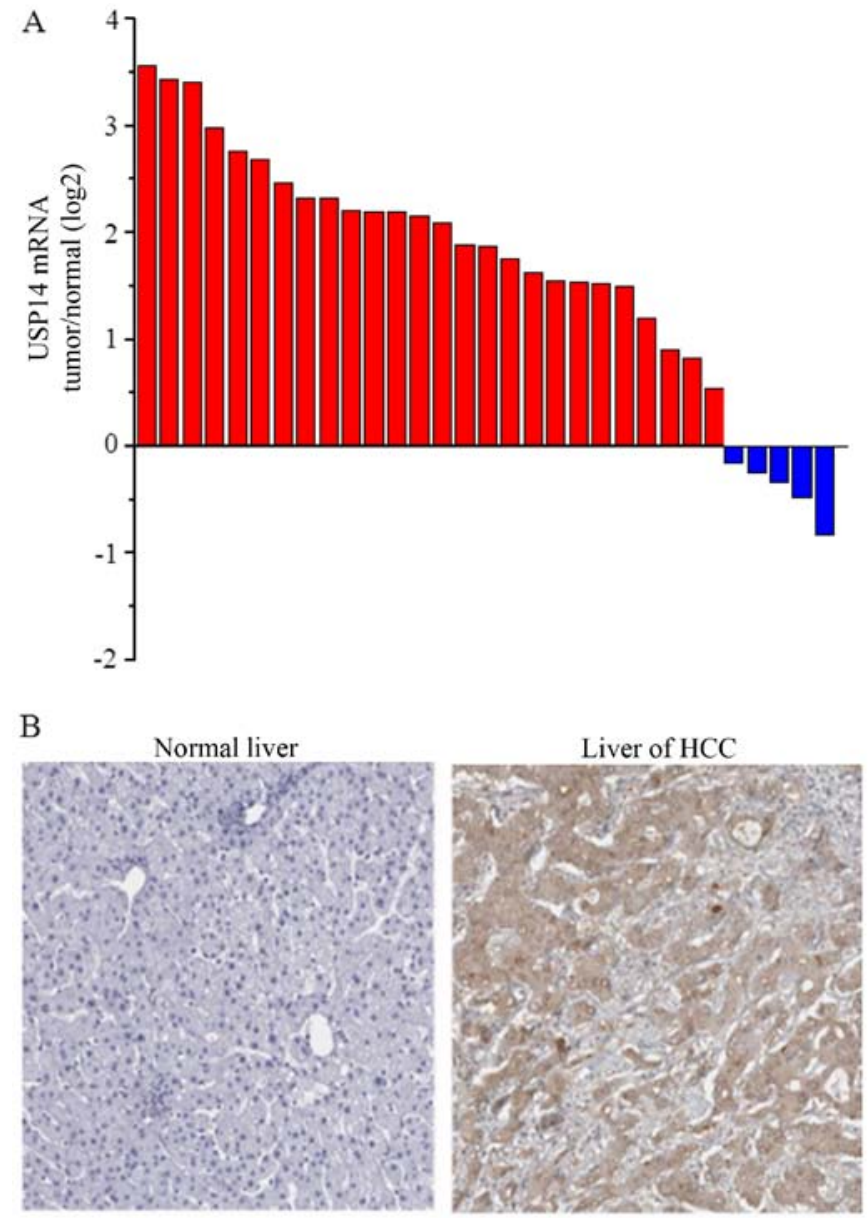

Figure 1. USP14 is overexpressed in human primary HCC tumor tissues. (A) Quantitative RT-PCR analysis for USP14 mRNA expression in 31 cases of HCC specimens. (B) Immunohistochemical analysis of USP14 protein expression in HCC and normal liver tissues. HCC, hepatocellular carcinoma.

intratumoral USP14 protein density. No significant correlation was detected between USP14 expression and the clinicopathological characteristics (Table I). No significant difference was detected in tumor size, tumor thrombus or sub foci incidence between the groups expressing high or low levels of USP14, although these parameters showed an increasing trend (Table I). However, there was a trend for progression of HCC in the USP14 high-expressing group. Of the 31 patients with HCC 26 were followed for 3 years. During the 3 -year follow-up period, 15 out of $26(57.69 \%)$ patients succumbed as a result of disease progression. The Kaplan-Meier curve analysis indicated that patients with a high USP14 expression (14 cases) had a significantly shorter overall survival rate $(35.71, \mathrm{P}<0.05)$ than those with a low USP14 expression (12 cases) (35.71 vs. $50 \%, \mathrm{P}<0.05$ ) (Fig. 2).

Silencing USP14 impairs HCC cell growth in vitro and in vivo. The expression of the USP14 protein was detected in seven HCC cell lines. The level of USP14 protein expression was markedly upregulated in the HepGB, HepG2, SMMC7721 and MHCC-97-L cell lines (Fig. 3A). We also detected the expression of USP14 mRNA in these seven HCC cell lines. The relative expression levels of the USP14 mRNAs were similar to those of the protein (Fig. 3B), with USP14 expres- 
Table I. Relationship between USP14 protein expression and HCC characteristics in training set $(n=26)$.

\begin{tabular}{lcrrr}
\hline & & \multicolumn{3}{c}{ USP 14 } \\
\cline { 3 - 4 } Characteristics & No. of patients (\%) & Low (n=12) & High (n=14) & P-value \\
\hline Age (years) & & $50.87 \pm 10.1$ & $49.7 \pm 11.9$ & 0.576 \\
Gender & & & & 0.657 \\
Male & $21(80.77)$ & 10 & 11 & \\
Female & $5(19.23)$ & 2 & 3 & 0.417 \\
HBV & & & & \\
Negative & $19(73.08)$ & $9(75)$ & $10(71.43)$ & 0.949 \\
Positive & $7(26.92)$ & $3(25)$ & $4(28.57)$ & 0.206 \\
AFP (ng/ml) & & 65.9 & 55.7 & 0.078 \\
Tumor size (cm) & $4.8 \pm 1.9$ & $5.2 \pm 1.7$ & 0.258 \\
Sub foci & $4(15.38)$ & $1(8.33)$ & $3(21.43)$ & $2(14.29)$ \\
Tumor thrombus & $3(11.54)$ & $1(8.33)$ & & \\
\hline
\end{tabular}

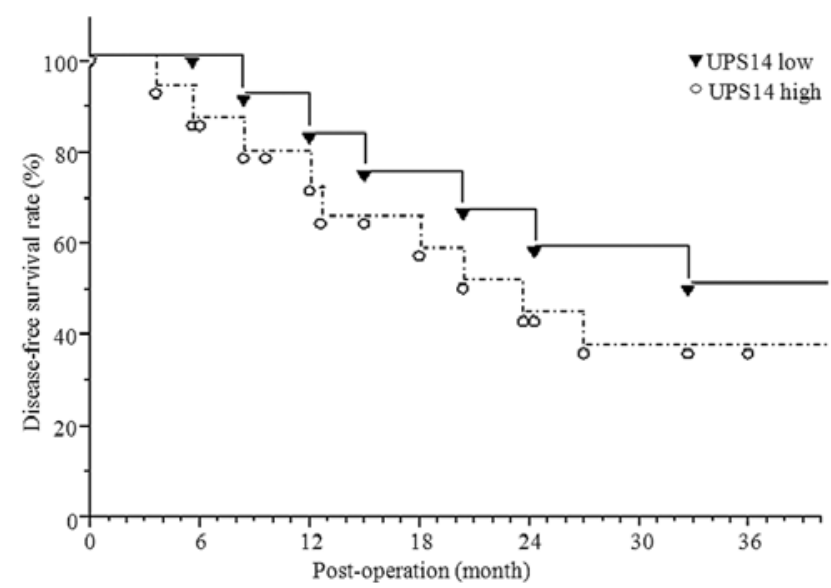

Figure 2. Kaplan-Meier plot of overall survival rate in patients with human primary HCC post-operation with high or low intratumoral USP14 expression. The DFS rate was analyzed in the high and low intratumoral USP14-expressing groups. The different subgroups are plotted according to their USP14 scores. HCC, hepatocellular carcinoma; DFS, disease-free survival.

sion being highest in SMMC7721 cells (Fig. 3). Therefore, SMMC7721 cells were selected for the transfection of three USP14 shRNA lentiviruses (USP14-siRNA1, USP14-siRNA2 and USP14-siRNA3). The USP14-siRNA2 significantly suppressed USP14 expression levels (Fig. 4A). The analyses of the cell growth curves under USP14 silencing conditions indicated that the proliferation of USP14-siRNA2 cells was significantly decreased compared with that in the control cells on days 4 and 5 ( $\mathrm{P}<0.01$ for both) (Fig. 4B).

We assessed the effects of USP14 depletion on colony formation in the SMMC7721 cell line (Fig. 4C and D). The depletion of USP14 markedly reduced colony formation in SMMC7721 cells. We also identified the role of USP14 in the SMMC7721 cell cycle using a FACS assay. The results demonstrated that the cell number in the $S$ phase was significantly decreased $(\mathrm{P}<0.05)$, while the cell number in $\mathrm{G} 0 / \mathrm{G} 1$ phase was significantly increased $(\mathrm{P}<0.05)$ after transfection with the USP14-shRNA lentivirus (Fig. 4E). We also examined the cells for apoptosis using Annexin V staining. We found that apoptosis in SMMC7721 cells was markedly increased after USP14-RNAi-2 knockdown compared with that in the control cells (Fig. 4F), demonstrating that the cell survival rate was significantly reduced when USP14 was downregulated.

USP14 induces HCC by increasing $\beta$-catenin levels. USP14 activates the $\mathrm{Wnt} / \beta$-catenin signaling pathway through deubiquitination (33). In A549 cancer cells, $\beta$-catenin protein levels were decreased following USP14 knockdown (28). Thus, we examined the expression profile of $\beta$-catenin in seven patients with HCC. Compared with normal liver tissues, the expression level of $\beta$-catenin was increased in HCC patients with high USP14 expression levels. The target genes of $\beta$-catenin (such as c-myc, axin 2 and cyclin D) were also increased in these patients (Fig. 5).

\section{Discussion}

HCC is a common malignant disease with high rates of metastasis and relapse. The mortality rate ranks third in the world and second in China (4), where $>100,000$ people succumb annually from HCC. The early diagnosis of HCC is difficult, with most cases undetected until the advanced stages (6). No satisfactory treatment for advanced HCC is currently available (34). Therefore, a new target therapy in HCC is required.

In the present study, we reported that the expression of USP14 was significantly higher in HCC than in normal liver tissues. We also analyzed the prognostic value of USP14 in HCC. In the present study, we examined the correlation between the molecular features of USP14 and clinical outcomes in HCC. We found no significant difference in tumor size between the groups expressing high or low levels of USP14. However, the group with a high USP14 expression showed a greater disease progression. The Kaplan-Meier curve analysis indicated that patients with a high USP14 expression (14 cases) had a significantly shorter overall 


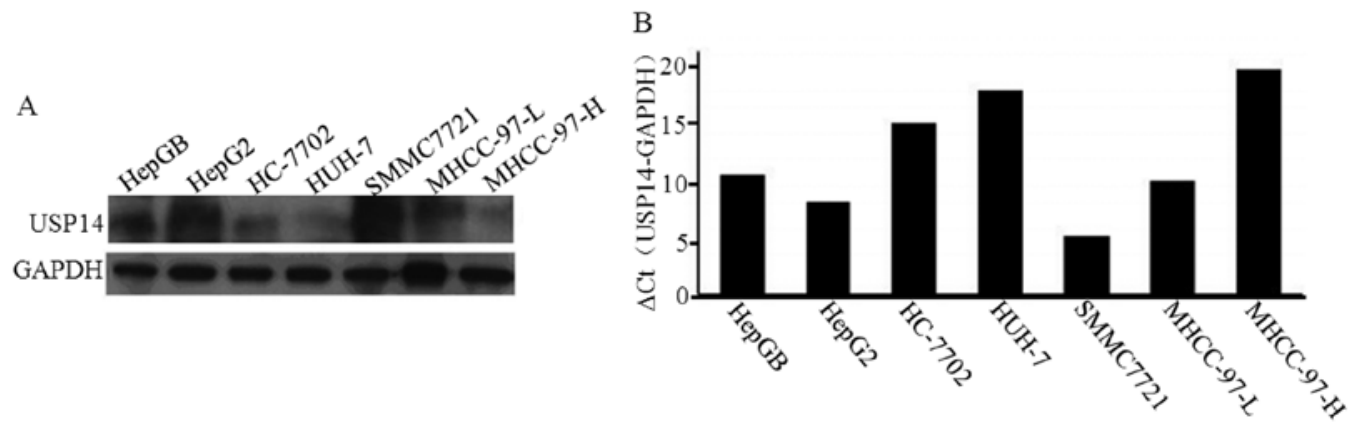

Figure 3. Expression levels of USP14 in human primary HCC cell lines. (A) Western blots showing the expression level of USP14 protein in the HCC cell lines. GAPDH is used as the internal control. (B) Quantitative RT-PCR results for USP14 mRNA levels in the HCC cell lines. HCC, hepatocellular carcinoma.
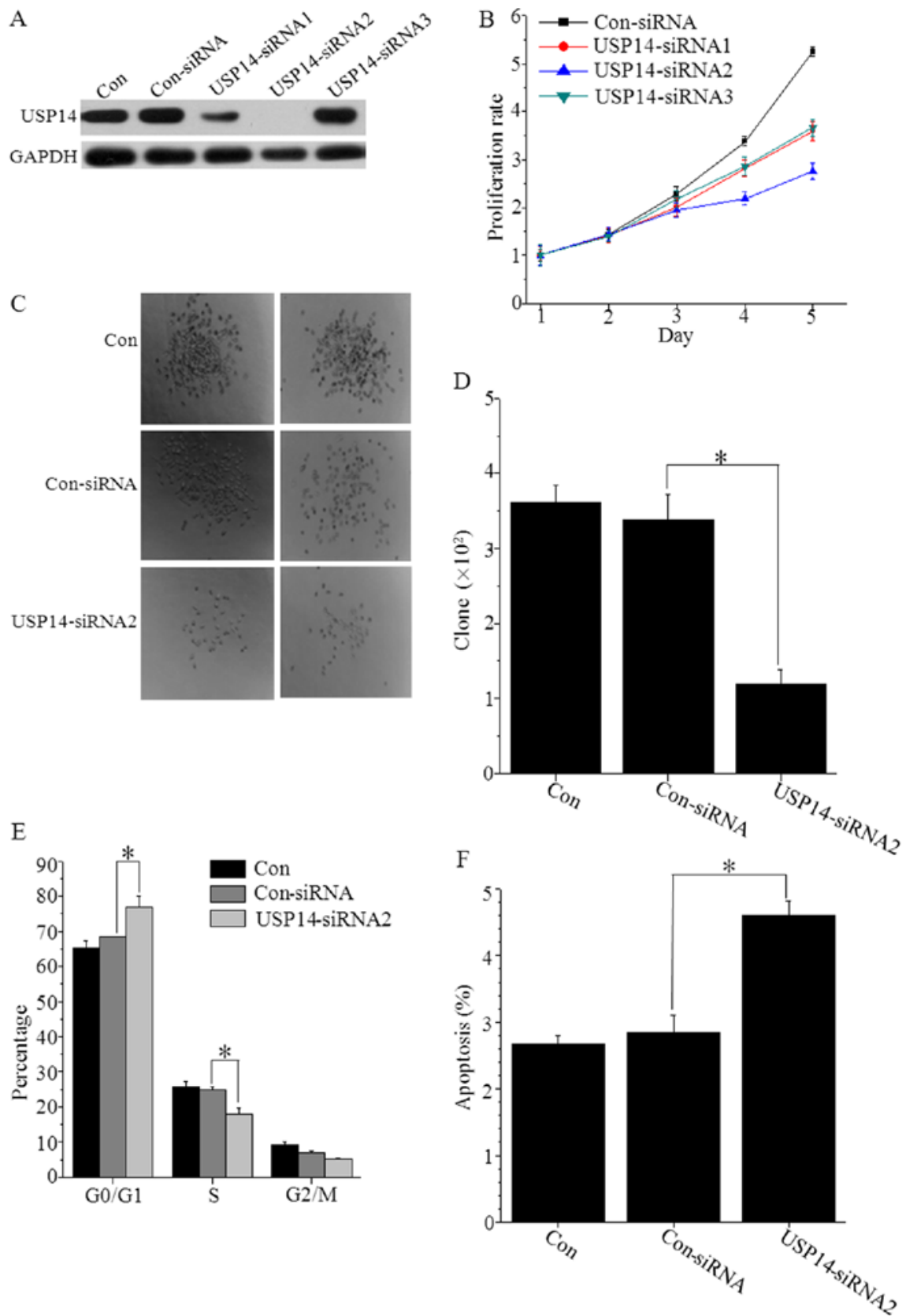

Figure 4. USP14 silencing impairs human SMMC7721 hepatocarcinoma cell growth. (A) Western blot analysis of the knockdown efficiency of USP14. (B) SMMC7721 cell growth curves after silencing USP14 as assessed with a CCK-8 assay. (C and D) Clonogenic assay results in the SMMC7721 cell line after USP14 knockdown with lentivirus-delivered siRNA. (E) Percentage of SMMC7721 cells in G0/G1, S and G2/M phases with various treatments. After USP14 silencing, the percentage of SMMC7721 cells in the G0/G1 phase increased, while the percentage of cells in the S phase decreased compared with that in the control cells. ${ }^{*} \mathrm{P}<0.05$. (F) Inhibition of USP14 expression-induced apoptosis in SMMC7721 cells. ${ }^{*} \mathrm{P}<0.05$. 
A
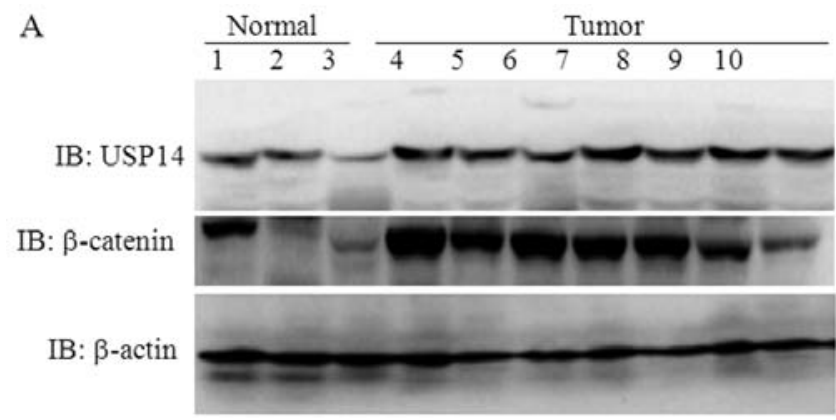

B

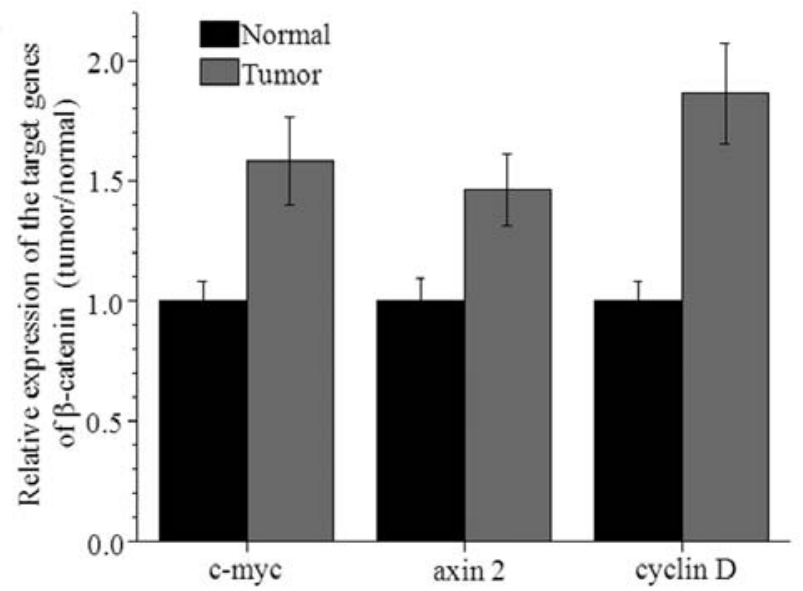

Figure 5. Increasing $\beta$-catenin levels in hepatocellular carcinoma patients with high USP14 expression levels. (A) Comparison of USP14 protein levels in normal $(n=3)$ and tumorous $(n=7)$ human liver tissues. (B) The expression levels of c-myc, axin 2 and cyclin D1 in 7 human HCC tissues and 3 normal livers. HCC, hepatocellular carcinoma.

survival rate $(35.71, \mathrm{P}<0.05)$ than those with a low USP14 expression (12 cases) (35.71 vs. $50 \%, \mathrm{P}<0.05)$.

USP14 is highly expressed in some tumors. Ishiwata et al were the first to study the upregulation of USP14 expression in leukemia cells (35). The high expression of USP14 was also found in a variety of colon cancer cells and in colorectal tissues of patients with colorectal cancer $(25,26)$. The clinical prognosis in patients with colorectal cancer is poor when USP14 expression is high. The expression of USP14 in colorectal cancer is strong and positively correlated with liver or lymph node metastasis or both. The mechanism of this metastasis may be associated with the regulation of tumor infiltration mediated by metastasis-associated protein MMP9 (26). Chuensumran et al (29) reported that pathological grading was directly associated with the expression level of USP14 in patients with intrahepatic cholangiocarcinoma. Another study using retroviral expression library screening indicated that USP14 may be important in the occurrence of ovarian cancer (27).

The present study found that USP14 was highly expressed in the SMMC7721 cell line. The growth of SMMC7721 cells was markedly decreased in vitro after knocking down the expression of USP14 with USP14-siRNA. After USP14 silencing, the suppression of SMMC7721 cell proliferation was $>50 \%$, the cell cycle was clearly altered and the apoptotic ratio was greatly increased. In addition, the ability of the SMMC7721 cells to form colonies was significantly inhibited. D'Arcy et al reported that b-AP15, a specific inhibitor of
USP14 and HCHL5, effectively inhibits a variety of allogeneic transplantation tumor growths (36). The inhibitor b-AP15 activates caspase- 3 in the cytosol, leading to the downstream accumulation in the cytosol of P53, CDKN1A and CDKN1B. Lee et al found that this specific small molecule inhibitor of USP14 increased the activity of proteasomes by promoting the ubiquitination of its substrate protein cyclin B to effectively inhibit allogeneic transplantation tumor growth (37). Wu et al reported that the overexpression of USP14 was highly associated with poor prognosis in patients with non-small cell lung cancer (NSCLC) (28). The high expression of USP14 in patients with NSCLC promoted tumor cell proliferation through the accumulation of $\beta$-catenin. USP14 regulates cell proliferation and apoptosis in epithelial ovarian cancer (27). Auranofin, a clinically used antirheumatic agent, is a proteasomal deubiquitinase inhibitor that inhibits tumor growth $(38,39)$.

Previous findings have shown that a deubiquitinating enzyme influenced the stability of cell cycle-associated proteins to regulate the cell cycle and this was closely associated with the occurrence and development of tumors (40). Many cell cycle-related proteins (such as cyclin A, D, B, E and cyclin-dependent kinases) play vital roles in regulating the transformation process. Their dysregulation can lead to the occurrence and development of many tumors (41-46). The intracellular half-life of these cell cycle-related proteins is short because they are normally selectively degraded by proteasomes following ubiquitination. Thus, with the overexpression of DUBs, ubiquitination-dependent degradation may be inhibited, leading to the accumulation of cell cycle-related proteins in cells. USP9X can stabilize myeloid cell leukemin-1 to promote the growth of tumor cells (22). The USP28 protein inhibited the ubiquitination activity of Fbxw7 ligase to stabilize cyclin-E1 and c-Myc protein in patients with colon or breast cancer, in which the USP14 protein was overexpressed (23). USP44 protein was also shown to inhibit the activity of the anaphase-promoting protein APC/C via removal of the effects of the ubiquitin modification effect of $\mathrm{Cdc} 20$ to prevent premature failure of the spindle checkpoint (24).

The ubiquitin proteasome system is a major intracellular protein degradation pathway involved in the regulation of the cell cycle, immune response, signal transduction and DNA repair in eukaryotic cells. The DUBs function to reverse the process of the UPS, ensuring a dynamic balance in the degradation of proteins. The UPS is strictly regulated to maintain protein homeostasis in cells. Thus, the dysregulation of the UPS pathway may induce a variety of diseases, including cancer. The UPS has proven to be an important target for cancer treatment. Many studies have screened compounds or drugs based on their ability to inhibit proteasome activity. For example, bortezomib, a drug targeting the ubiquitin-proteasome pathway, was approved for the treatment of multiple myeloma (MM) and mantle cell lymphoma (MCL) by the Food and Drug Administration (FDA) over 12 years ago (47). Subsequently, carfilzomib, another drug targeting the ubiquitin-proteasome pathway, was approved by the FDA for the treatment of patients with relapsed and refractory $\mathrm{MM}$ who received prior bortezomib and lenalidomide or thalidomide (48). Thousands of patients suffering from myeloma or lymphoma have benefited from bortezomib- or carfilzomib-based therapy and the overall 
survival rate of MM has been significantly increased in the last decade (48-50). In addition, copper pyrithione, a potent inhibitor of proteasome-specific UCHL5 and USP14, inhibits tumor growth in vivo (51).

In the present study we detected the expression profile of USP14 during the progression of HCC, and analyzed the relationship between USP14 and the malignant transformation of hepatocytes. Furthermore, we found that USP14 promoted HCC development by increasing HCC cell proliferation, altering the cell cycle and reducing apoptosis. These results indicate that USP14 may be a novel therapeutic target for preventing or combating $\mathrm{HCC}$, as well as a novel target for use in the clinical diagnosis or prognostic analysis of HCC to assist in designing personalized therapies for patients. The present study also provides evidence for the mechanism of HCC progression.

\section{Acknowledgements}

The present study was supported by grants awarded by the National Natural Science Foundation of China (no. 81201555), the Major State Basic Research Development Program of China (973 Program; no. 2014CB542102), the Science Fund for Creative Research Groups of the National Natural Science Foundation of China (no. 81201940) and the State Key Infectious Disease Project of China (nos. 2012ZX10002010 and 2012ZX10002016).

\section{References}

1. Kamangar F, Dores GM and Anderson WF: Patterns of cancer incidence, mortality, and prevalence across five continents: Defining priorities to reduce cancer disparities in different geographic regions of the world. J Clin Oncol 24: 2137-2150, 2006.

2. El-Serag HB and Rudolph KL: Hepatocellular carcinoma: Epidemiology and molecular carcinogenesis. Gastroenterology 132: 2557-2576, 2007.

3. El-Serag HB: Epidemiology of viral hepatitis and hepatocellular carcinoma. Gastroenterology 142:1264-1273, 2012.

4. Gao J, Xie L, Yang WS, Zhang W, Gao S, Wang J and Xiang YB: Risk factors of hepatocellular carcinoma - current status and perspectives. Asian Pac J Cancer Prev 13: 743-752, 2012.

5. Jemal A, Murray T, Ward E, Samuels A, Tiwari RC, Ghafoor A, Feuer EJ and Thun MJ: Cancer statistics, 2005. CA Cancer J Clin 55: 10-30, 2005.

6. Sakamoto M: Early HCC: Diagnosis and molecular markers. J Gastroenterol 44 (Suppl 19): 108-111, 2009.

7. Portolani N, Coniglio A, Ghidoni S, Giovanelli M, Benetti A, Tiberio GA and Giulini SM: Early and late recurrence after liver resection for hepatocellular carcinoma: Prognostic and therapeutic implications. Ann Surg 243: 229-235, 2006.

8. Giordano S and Columbano A: Met as a therapeutic target in HCC: Facts and hopes. J Hepatol 60: 442-452, 2014.

9. Llovet JM and Bruix J: Molecular targeted therapies in hepatocellular carcinoma. Hepatology 48: 1312-1327, 2008.

10. Newell P, Villanueva A and Llovet JM: Molecular targeted therapies in hepatocellular carcinoma: From pre-clinical models to clinical trials. J Hepatol 49: 1-5, 2008.

11. Azoulay D: Resection for hepatocellular carcinoma with hepatic vein tumour thrombus: Pushing the limits beyond the guidelines frontiers. J Hepatol 61: 462-463, 2014.

12. Cucchetti A, Djulbegovic B, Tsalatsanis A, Vitale A, Hozo I, Piscaglia F, Cescon M, Ercolani G, Tuci F, Cillo U, et al: When to perform hepatic resection for intermediate-stage hepatocellular carcinoma. Hepatology 61: 905-914, 2015.

13. Borodovsky A, Kessler BM, Casagrande R, Overkleeft HS Wilkinson KD and Ploegh HL: A novel active site-directed probe specific for deubiquitylating enzymes reveals proteasome association of USP14. EMBO J 20: 5187-5196, 2001.
14. Hu M, Li P, Song L, Jeffrey PD, Chenova TA, Wilkinson KD, Cohen RE and Shi Y: Structure and mechanisms of the proteasome-associated deubiquitinating enzyme USP14. EMBO J 24: 3747-3756, 2005

15. Peth A, Besche HC and Goldberg AL: Ubiquitinated proteins activate the proteasome by binding to Usp14/Ubp6, which causes 20S gate opening. Mol Cell 36: 794-804, 2009.

16. Glickman $\mathrm{MH}$ and Ciechanover A: The ubiquitin-proteasome proteolytic pathway: Destruction for the sake of construction. Physiol Rev 82: 373-428, 2002.

17. Kimura Y, Yashiroda H, Kudo T, Koitabashi S, Murata S, Kakizuka A and Tanaka K: An inhibitor of a deubiquitinating enzyme regulates ubiquitin homeostasis. Cell 137: 549-559, 2009.

18. Love KR, Catic A, Schlieker C and Ploegh HL: Mechanisms, biology and inhibitors of deubiquitinating enzymes. Nat Chem Biol 3: 697-705, 2007.

19. Sheaff RJ, Singer JD, Swanger J, Smitherman M, Roberts JM and Clurman BE: Proteasomal turnover of p21Cip1 does not require p21Cip1 ubiquitination. Mol Cell 5: 403-410, 2000.

20. Maki CG, Huibregtse JM and Howley PM: In vivo ubiquitination and proteasome-mediated degradation of p53(1). Cancer Res 56: 2649-2654, 1996.

21. Huang TT, Nijman SM, Mirchandani KD, Galardy PJ, Cohn MA, Haas W, Gygi SP, Ploegh HL, Bernards R and D'Andrea AD: Regulation of monoubiquitinated PCNA by DUB autocleavage. Nat Cell Biol 8: 339-347, 2006.

22. Schwickart M, Huang X, Lill JR, Liu J, Ferrando R, French DM, Maecker H, O'Rourke K, Bazan F, Eastham-Anderson J, et al: Deubiquitinase USP9X stabilizes MCL1 and promotes tumour cell survival. Nature 463: 103-107, 2010.

23. Popov N, Wanzel M, Madiredjo M, Zhang D, Beijersbergen R, Bernards R, Moll R, Elledge SJ and Eilers M: The ubiquitinspecific protease USP28 is required for MYC stability. Nat Cell Biol 9: 765-774, 2007.

24. Kim AH, Puram SV, Bilimoria PM, Ikeuchi Y, Keough S, Wong M, Rowitch D and Bonni A: A centrosomal Cdc20-APC pathway controls dendrite morphogenesis in postmitotic neurons. Cell 136: 322-336, 2009.

25. Ishiwata S, Ozawa Y, Katayama J, Kaneko S, Shindo H, Tomioka Y, Ishiwata T, Asano G, Ikegawa S and Mizugaki M: Elevated expression level of $60-\mathrm{kDa}$ subunit of tRNA-guanine transglycosylase in colon cancer. Cancer Lett 212: 113-119, 2004.

26. Shinji S, Naito Z, Ishiwata S, Ishiwata T, Tanaka N, Furukawa K, Suzuki H, Seya T, Matsuda A, Katsuta M, et al: Ubiquitin-specific protease 14 expression in colorectal cancer is associated with liver and lymph node metastases. Oncol Rep 15: 539-543, 2006.

27. Wada T, Yamashita Y, Saga Y, Takahashi K, Koinuma K, Choi YL, Kaneda R, Fujiwara S, Soda M, Watanabe H, et al: Screening for genetic abnormalities involved in ovarian carcinogenesis using retroviral expression libraries. Int J Oncol 35: 973-976, 2009.

28. Wu N, Liu C, Bai C, Han YP, Cho WC and Li Q: Over-expression of deubiquitinating enzyme USP14 in lung adenocarcinoma promotes proliferation through the accumulation of $\beta$-catenin. Int J Mol Sci 14: 10749-10760, 2013.

29. Chuensumran U, Saelee P, Punyarit P, Wongkham S, Pairojkul C, Chauin S and Petmitr S: Ubiquitin-specific protease 14 expression associated with intrahepatic cholangiocarcinoma cell differentiation. Asian Pac J Cancer Prev 12: 775-779, 2011.

30. Zhang C, Ling Y, Zhang C, Xu Y, Gao L, Li R, Zhu J, Fan L and Wei L: The silencing of RECK gene is associated with promoter hypermethylation and poor survival in hepatocellular carcinoma. Int J Biol Sci 8: 451-458, 2012.

31. Dong LW, Hou YJ, Tan YX, Tang L, Pan YF, Wang M and Wang HY: Prognostic significance of Beclin 1 in intrahepatic cholangiocellular carcinoma. Autophagy 7: 1222-1229, 2011.

32. Wang Q, Tan YX, Ren YB, Dong LW, Xie ZF, Tang L, Cao D, Zhang WP, Hu HP and Wang HY: Zinc finger protein ZBTB20 expression is increased in hepatocellular carcinoma and associated with poor prognosis. BMC Cancer 11: 271, 2011.

33. Jung H, Kim BG, Han WH, Lee JH, Cho JY, Park WS, Maurice MM, Han JK, Lee MJ, Finley D, et al: Deubiquitination of Dishevelled by Usp14 is required for Wnt signaling. Oncogenesis 2: e64, 2013.

34. Germano D and Daniele B: Systemic therapy of hepatocellular carcinoma: Current status and future perspectives. World J Gastroenterol 20: 3087-3099, 2014.

35. Ishiwata S, Katayama J, Shindo H, Ozawa Y, Itoh K and Mizugaki M: Increased expression of queuosine synthesizing enzyme, tRNA-guanine transglycosylase, and queuosine levels in tRNA of leukemic cells. J Biochem 129: 13-17, 2001. 
36. D'Arcy P, Brnjic S, Olofsson MH, Fryknäs M, Lindsten K, De Cesare M, Perego P, Sadeghi B, Hassan M, Larsson R, et al: Inhibition of proteasome deubiquitinating activity as a new cancer therapy. Nat Med 17: 1636-1640, 2011.

37. Lee BH, Lee MJ, Park S, Oh DC, Elsasser S, Chen PC, Gartner C, Dimova N, Hanna J, Gygi SP, et al: Enhancement of proteasome activity by a small-molecule inhibitor of USP14. Nature 467: 179-184, 2010

38. Liu N, Li X, Huang H, Zhao C, Liao S, Yang C, Liu S, Song W, Lu X, Lan X, et al: Clinically used antirheumatic agent auranofin is a proteasomal deubiquitinase inhibitor and inhibits tumor growth. Oncotarget 5: 5453-5471, 2014.

39. Chen X, Shi X, Zhao C, Li X, Lan X, Liu S, Huang H, Liu N, Liao S, Zang D, et al: Anti-rheumatic agent auranofin induced apoptosis in chronic myeloid leukemia cells resistant to imatinib through both Bcr/Abl-dependent and -independent mechanisms. Oncotarget 5: 9118-9132, 2014.

40. Nakayama KI, Nakayama K: Ubiquitin ligases: cell-cycle control and cancer. Nat Rev cancer 6: 369-381, 2006.

41. Husdal A, Bukholm G and Bukholm IR: The prognostic value and overexpression of cyclin A is correlated with gene amplification of both cyclin A and cyclin $\mathrm{E}$ in breast cancer patient. Cell Oncol 28: 107-116, 2006.

42. Nauman A, Turowska O, Poplawski P, Master A, Tanski Z and Puzianowska-Kuznicka M: Elevated cyclin E level in human clear cell renal cell carcinoma: Possible causes and consequences. Acta Biochim Pol 54: 595-602, 2007.

43. Chang KC, Chang Y, Jones D and Su IJ: Aberrant expression of cyclin a correlates with morphogenesis of reed-sternberg cells in Hodgkin lymphoma. Am J Clin Pathol 132: 50-59, 2009.
44. Cooley A, Zelivianski S and Jeruss JS: Impact of cyclin E overexpression on Smad3 activity in breast cancer cell lines. Cell Cycle 9: 4900-4907, 2010.

45. Wang LH, Huang W, Lai MD and Su IJ: Aberrant cyclin A expression and centrosome overduplication induced by hepatitis B virus pre-S2 mutants and its implication in hepatocarcinogenesis. Carcinogenesis 33: 466-472, 2012.

46. Freije A, Ceballos L, Coisy M, Barnes L, Rosa M, De Diego E, Blanchard JM and Gandarillas A: Cyclin E drives human keratinocyte growth into differentiation. Oncogene 31: 5180-5192, 2012.

47. Richardson PG, Barlogie B, Berenson J, Singhal S, Jagannath S, Irwin D, Rajkumar SV, Srkalovic G, Alsina M, Alexanian R, et al: A phase 2 study of bortezomib in relapsed, refractory myeloma. N Engl J Med 348: 2609-2617, 2003.

48. Thompson JL: Carfilzomib: A second-generation proteasome inhibitor for the treatment of relapsed and refractory multiple myeloma. Ann Pharmacother 47: 56-62, 2013.

49. Richardson PG, Mitsiades C, Hideshima T and Anderson KC: Bortezomib: Proteasome inhibition as an effective anticancer therapy. Annu Rev Med 57: 33-47, 2006.

50. Cavo M: Proteasome inhibitor bortezomib for the treatment of multiple myeloma. Leukemia 20: 1341-1352, 2006.

51. Liu N, Liu C, Li X, Liao S, Song W, Yang C, Zhao C, Huang H, Guan L, Zhang P, et al: A novel proteasome inhibitor suppresses tumor growth via targeting both $19 \mathrm{~S}$ proteasome deubiquitinases and 20S proteolytic peptidases. Sci Rep 4: 5240, 2014. 